\title{
Estudos de algumas correlações dos ovários com os corpos lúteos e o desenvolvimento fetal em fêmeas de bovinos Nelore
}

\author{
Renata Barbieri Trevisan ${ }^{1}$ \\ Wilson Machado de Souza ${ }^{2 *}$ \\ ${ }^{1}$ Faculdade de Zootecnia e Engenharia de Alimentos, Universidade de São Paulo \\ Rua Antônio Magnani, 639, Jd. Eldorado, CEP 13630-310, Pirassununga - SP, Brasil \\ ${ }^{2}$ Faculdade de Medicina Veterinária, Departamento de Apoio, Produção e Saúde Animal \\ Universidade Estadual Paulista, Campus Araçatuba \\ Rua Clovis Pestana, 793, Bairro Dona Amélia, CEP 16050-680, Araçatuba - SP, Brasil \\ * Autor para correspondência \\ souzawm@fmva.unesp.br
}

Submetido em 22/07/2011

Aceito para publicação em 09/01/2012

\section{Resumo}

Foram avaliados os ovários direitos e esquerdos de 30 vacas prenhes da raça Nelore, cujo aparelhos reprodutores foram coletados em frigoríficos da região de Oeste do Estado de São Paulo. Este material foi conduzido ao Laboratório de Anatomia Animal do campus da Unesp de Araçatuba, para serem analisados o tamanho do feto, por meio de medida occipito-sacral, sua altura e peso. Também foram obtidos dos ovários e corpos lúteos o comprimento, largura e espessura por meio de paquímetro. Destas gônadas foram analisados também o seu peso e volume. Estas informações relativas aos ovários e aos corpos lúteos foram correlacionadas com o desenvolvimento fetal, utilizando o programa SAS para analisar o coeficiente de correlação de Pearson, além de ajustado um modelo de Regressão Linear Simples. Verificou-se que houve correlação significativa entre as variáveis ovários direitos e desenvolvimento fetal, positiva para largura e negativa para espessura. Já entre o corpo lúteo e desenvolvimento do feto houve correlação significativa negativa para o volume.

Palavras-chave: Aparelho reprodutor; Feto; Morfologia

\section{Abstract}

Studies of some correlations of ovaries with corpora lutea and fetal development in female Nelore cows. This study evaluated the right and left ovaries of 30 pregnant Nelore cows. The reproductive tracts were collected at slaughterhouses in the western region of the state of São Paulo, and the study was conducted at the Laboratório de Anatomia Animal at UNESP (in Araçatuba). The size (occipitosacral length), height and weight of the fetuses were analyzed. The length, width and thickness (using calipers) of the ovaries and corpora lutea, and weight and volume of the gonads were also measured. The data related to the ovaries and corpora lutea were correlated with fetal development, using a SAS program to analyze the Pearson correlation coefficient, and adjusted using a simple linear regression model. There was a significant correlation between the right ovary variables and fetal development; positive for width and negative for thickness. For the corpora lutea and fetal development, there was a significant negative correlation with the volume.

Key words: Fetus; Morphology; Reproductive System 


\section{Introdução}

Das raças zebuínas, a Nelore é a mais criada no Brasil, gerando consideráveis recursos econômicos, especialmente nas regiões Sudeste e Centro-Oeste.

Evidentemente, com o avanço da cultura da canade-açúcar em praticamente todas as regiões do estado de São Paulo, a criação e exploração destes animais tende a ficar restrita e especializar-se, já que a viabilidade econômica da produção de bovinos de corte no Brasil está diretamente relacionada ao sistema de produção adotado. Com a especialização da produção, o papel da reprodução animal torna-se fundamental e com isso há necessidade de conhecimentos cada vez mais profundos da anatomofisiologia do aparelho reprodutor.

Este aparelho sofre acentuadas alterações morfológicas durante a vida reprodutiva das fêmeas, destacando-se aquelas que ocorrem durante o ciclo estral. Particularizando as gônadas femininas, os ovários são órgãos pares, cuja forma e a posição são variáveis de acordo com a espécie animal. Nestes órgãos, os corpos lúteos são estruturas marcantes que se projetam na sua superfície em menor ou maior tamanho na dependência da fase do ciclo reprodutivo, bem como durante o período gestacional.

A palpação retal, apesar das suas limitações, ainda é um método bastante utilizado na prática para diagnóstico da gestação e avaliação direta dos ovários. Entretanto, a interpretação dos achados pode ser dificultada pelas diversas formas e volumes destes órgãos. Tais alterações morfológicas podem ser explicadas pelo dinamismo das funções ovarianas.

Neste contexto, este trabalho teve como objetivo estabelecer uma análise morfológica e morfométrica dos ovários e corpos lúteos, correlacionando estes dados com a fase do desenvolvimento fetal, e com isto facilitar a interpretação dos achados clínicos no exame ginecológico e ajudar na contribuição de novos dados para futuras pesquisas com bovinos da raça Nelore.

\section{Material e Métodos}

Neste estudo foram utilizadas 30 peças retiradas de fêmeas de bovino da raça Nelore, adultas e prenhes em diferentes fases da gestação, com preferência do primeiro terço gestacional. Estes animais são procedentes da região de Araçatuba e abatidos em frigorífico de Guararapes, Estado de São Paulo.

O trato genital destas vacas foi coletado em bloco e conduzido ao laboratório de Anatomia Animal, do Departamento de Apoio, Produção e Saúde Animal do campus UNESP de Araçatuba. Depois de lavado em água corrente, o corno uterino gestante foi aberto, assim como as membranas componentes da placenta. Logo após, foi isolado o feto e seccionado o funículo umbilical Além da determinação do sexo destes animais, foram tomadas as medidas occipito-sacral (MOS), conforme as recomendações de Roberts (1986), a altura, com auxílio de uma fita métrica, e o peso dos mesmos.

Simultaneamente os ovários direitos e esquerdos foram isolados, procedendo-se a sua pesagem e determinação do comprimento, largura e espessura, anotando-se ainda a presença do corpo lúteo gestante. Estes foram isolados por dissecção cirúrgica para analisar seu comprimento, largura, espessura, peso, e imersão total no parênquima do ovário. As medidas destas duas estruturas (ovários e corpo lúteo) foram obtidas mediante a utilização de paquímetro. Tanto dos ovários quanto dos corpos lúteos foram obtidos por meio da imersão individual por deslocamento líquido em proveta graduada. Os resultados foram analisados estatisticamente, com o objetivo de estabelecer a correlação entre as medidas obtidas dos ovários e dos corpos lúteos com a fase do desenvolvimento fetal. Os termos utilizados na exposição deste trabalho estão de acordo com a Nomenclatura Anatômica Veterinária (SCHALLER, 1999).

As comparações estatísticas foram executadas com o uso do teste ANOVA. No caso de haver diferença significativa, foi aplicado o teste de Tukey. Todos os resultados foram expressos como média \pm erro padrão. Os valores de $\mathrm{p} \leq 0,05$ foram considerados para se determinar diferenças entre as médias. As correlações entre a morfologia das estruturas (ovários e corpos lúteos) e desenvolvimento fetal foram analisadas utilizando o método de Coeficiente de Correlação de Pearson. Todas as análises foram obtidas pelo programa estatístico SAS ${ }^{\circledR}$ (2002). 


\section{Resultados}

A média do desenvolvimento fetal, segundo sua medida occipito-sacral (MOS), foi de 26,33 $\pm 18,23 \mathrm{~cm}$.

Em relação às medidas de peso $(\mathrm{g})$, comprimento $(\mathrm{cm})$, largura $(\mathrm{cm})$, espessura $(\mathrm{cm})$ e volume $(\mathrm{ml})$ dos ovários, pode-se observar que os ovários direitos foram maiores que as medidas para ovários esquerdos em todas as amostras observadas (Tabela 1).

TABELA 1: Média \pm erro padrão das medidas dos ovários direitos e esquerdos.

\begin{tabular}{lcc}
\hline \multicolumn{3}{c}{ Ovários } \\
\hline Direito & Esquerdo \\
\hline Peso & $7,341 \pm 0,355 \mathrm{a}$ & $4,677 \pm 0,356 \mathrm{~b}$ \\
Comprimento & $3,284 \pm 0,076 \mathrm{a}$ & $2,859 \pm 0,076 \mathrm{~b}$ \\
Largura & $2,485 \pm 0,070 \mathrm{a}$ & $2,070 \pm 0,070 \mathrm{~b}$ \\
Espessura & $1,730 \pm 0,053 \mathrm{a}$ & $1,367 \pm 0,053 \mathrm{~b}$ \\
Volume & $7,650 \pm 0,370 \mathrm{a}$ & $4,700 \pm 0,370 \mathrm{~b}$ \\
\hline
\end{tabular}

Médias seguidas por letras distintas diferem entre si pelo Teste de Tukey $(\mathrm{p}<0,05)$.

Analisando o Coeficiente de Correlação de Pearson entre as variáveis dos ovários direitos e desenvolvimento do feto (Tabela 2), obteve-se uma correlação significativa para largura e espessura do ovário, onde a primeira foi positiva, ou seja, conforme aumenta o desenvolvimento do feto observou-se também um aumento da largura dos ovários. Já a segunda foi negativa, indicando que conforme aumenta o desenvolvimento fetal há uma diminuição da espessura dos ovários, todos com nível de $\mathrm{p}<0,05$.

Para a análise do Coeficiente de Correlação de Pearson entre as variáveis dos ovários esquerdos e desenvolvimento do feto (Tabela 2), não foi observada uma correlação significativa para nenhuma das variáveis, nos quais todas não significativas ao nível $p<0,05$. Já que o número de ovários esquerdos com a presença do corpo lúteo foi muito reduzido na amostra utilizada, isto pode ter influenciado na análise dos dados na estatística $(\mathrm{p}<0,05)$.

Para as medidas dos corpos lúteos, os direitos foram maiores que os esquerdos em todas as amostras (Tabela 3). Da correlação entre o corpo lúteo e a MOS (Tabela 4), somente o volume teve uma correlação significativa negativa, indicativo de que conforme há um aumento do tamanho do feto, observa-se uma diminuição do volume do corpo lúteo, com nível de $\mathrm{p}<0,05$.

TABELA 2: Coeficiente de Correlação de Pearson entre as variáveis medida occipito-sacral (MOS) e medidas dos ovários (peso, comprimento, largura, espessura e volume) direitos e esquerdos.

\begin{tabular}{lcccc}
\hline & \multicolumn{2}{c}{ Ovários } \\
\hline & $\mathbf{r}$ & $\mathbf{p}-$ valor & $\mathbf{r}$ & $\mathbf{p}-$ valor \\
\hline Peso & 0,055200 & $0,7933 \mathrm{NS}$ & 0,046993 & $0,9402 \mathrm{NS}$ \\
Comprimento & 0,343794 & $0,0924 \mathrm{NS}$ & 0,056231 & $0,9284 \mathrm{NS}$ \\
Largura & 0,452444 & $0,0232 *$ & $-0,520490$ & $0,3686 \mathrm{NS}$ \\
Espessura & $-0,543543$ & $0,0050 * *$ & 0,560794 & $0,3254 \mathrm{NS}$ \\
Volume & $-0,039947$ & $0,8496 \mathrm{NS}$ & 0,049855 & $0,9365 \mathrm{NS}$ \\
\hline
\end{tabular}

*Correlação positiva e significativa $(\mathrm{p}<0,05)$; **Correlação negativa e significativa $(\mathrm{p}<0,05)$. NS - Correlação não significativa. 
TABELA 3: Média \pm erro padrão das medidas dos corpos lúteos direitos e esquerdos.

\begin{tabular}{lcc}
\hline & Corpo Lúteo \\
\hline & Direito & Esquerdo \\
\hline Peso & $3,289 \pm 0,153 \mathrm{a}$ & $2,988 \pm 0,341 \mathrm{~b}$ \\
Comprimento & $2,200 \pm 0,048 \mathrm{a}$ & $2,132 \pm 0,108 \mathrm{~b}$ \\
Largura & $1,944 \pm 0,040 \mathrm{a}$ & $1,910 \pm 0,090 \mathrm{~b}$ \\
Espessura & $1,547 \pm 0,033 \mathrm{a}$ & $1,462 \pm 0,073 \mathrm{~b}$ \\
Volume & $3,360 \pm 0,215 \mathrm{a}$ & $3,000 \pm 0,480 \mathrm{~b}$ \\
\hline
\end{tabular}

Médias seguidas por letras distintas diferem entre si pelo Teste de Tukey $(\mathrm{p}<0,05)$.

As Figuras 1 e 2 representam as Regressões Lineares Simples dos pesos, comprimentos, larguras, espessuras e volumes dos ovários direitos e esquerdos e dos corpos lúteos, respectivamente.

TABELA 4: Coeficiente de Correlação de Pearson entre as variáveis medida occipito-sacral (MOS) e medidas dos corpos lúteos (peso, comprimento, largura, espessura e volume).

\begin{tabular}{lcc}
\hline \multicolumn{3}{c}{ Corpos Lúteos } \\
\hline & $\mathbf{r}$ & $\mathbf{p}-$ valores \\
\hline Peso & $-0,04356$ & $0,8192 \mathrm{NS}$ \\
Comprimento & 0,12591 & $0,5074 \mathrm{NS}$ \\
Largura & 0,09936 & $0,6014 \mathrm{NS}$ \\
Espessura & $-0,18976$ & $0,3152 \mathrm{NS}$ \\
Volume & $-0,37620$ & $0,0405 *$ \\
\hline
\end{tabular}

*Correlação negativa e significativa $(\mathrm{p}<0,05)$. NS - Correlação não significativa.

\section{Discussão}

O corpo lúteo, uma glândula endócrina transitória, durante a sua formação, manutenção e regressão, apresenta características morfológicas distintas (NEVES et al., 2002) e passa por intensa remodelação tecidual caracterizada por proliferação, diferenciação e morte celular. Após a ovulação, o corpo lúteo é formado a partir das células do folículo ovulatório em um processo conhecido como luteinização. Apresenta dois tipos de células esteroidogênicas e é uma estrutura primariamente reconhecida pela habilidade em sintetizar e secretar progesterona, hormônio que está intimamente relacionado com o estabelecimento e manutenção da gestação (FIELDS; FIELDS, 1996). Zheng et al. (1994) observou que o crescimento inicial do corpo lúteo é excerbado e seu peso aumenta mais de seis vezes na primeira metade do ciclo estral bovino, secretando quantidades crescentes de progesterona. Binelli (2000) relatou que o corpo lúteo atinge seu tamanho máximo aproximadamente no dia 10 do ciclo.

Os informes dos tratados de Anatomia Veterinária nos mostram dados genéricos sobre a fisiologia ovariana, parcimoniosos e por vezes contraditórios quando tratam do assunto. Além de certamente serem referentes ao gado taurino (europeu) desta forma, Getty (1986) esclarece que os ovários da vaca são menores que os ovários da égua $\mathrm{e}$ medem de 3,5 a 4,0 cm de comprimento, 2,5 de largura e 1,5 de espessura e o seu peso médio é de 15 a $20 \mathrm{~g}$. Possuem formato oval, são pontiagudos na extremidade uterina e não possuem fossa de ovulação. Normalmente estão situados próximo ao centro da margem lateral da entrada pélvica, cranialmente à atéria ilíaca externa na fêmea não grávida, mas podem estar mais cranialmente, especialmente nas vacas que já passaram por gravidez. Os dados acima, embora não se afastem das nossas observações em relação ao comprimento, a largura e a espessura, diferem quanto ao peso, sendo superiores aos nossos. Uma das possibilidades desta diferença pode ser decorrente das observações apresentadas pelo autor serem obtidas em bovinos de origem Europeia.

Schwarze e Schröder (1970) referem que, em animais uníparos, após a ovulação ocorre hemorragia na cavidade folicular, seguida de proliferação de células luteínicas, para compor o corpo lúteo. Este alcança, na vaca, até $3,0 \mathrm{~cm}$ de comprimento, valor este superior ao referido por Getty (1986), que foi de 1,0 a 1,5cm. Em nossos resultados referentes aos bovinos Nelore foram encontrados valores médios de $2,20 \mathrm{~cm}$, mais próximos dos encontrados por Schwarze e Schröder (1970).

Segundo Frandson (1979), os hormônios produzidos nos ovários incluem os estrógenos dos folículos e a progesterona dos corpos lúteos, que pelos informes do autor apresentam flutuações de acordo com a fase gestacional. Também quanto ao corpo lúteo, Nickel et al. (1979), Barone (1990), Dyce et al. (1997); e Konig e Liebich (2011) aludem que a prenhez determina a presença de um corpo lúteo marcante na superfície do ovário, cuja estrutura apresenta uma consistência 
FIGURA 1: Regressão Linear Simples dos pesos (A), comprimentos (B), larguras (C), espessuras (D) e volumes (E) dos ovários direitos e dos ovários esquerdos.

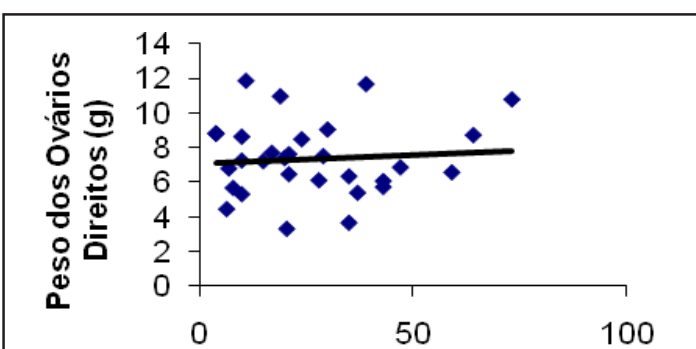

Medida Occipito-sacral dos Fetos $(\mathrm{cm})$

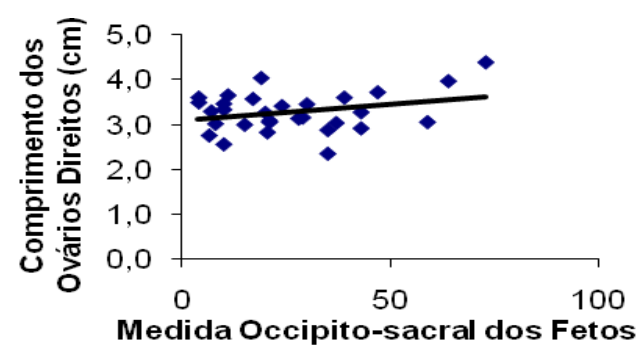
(cm)
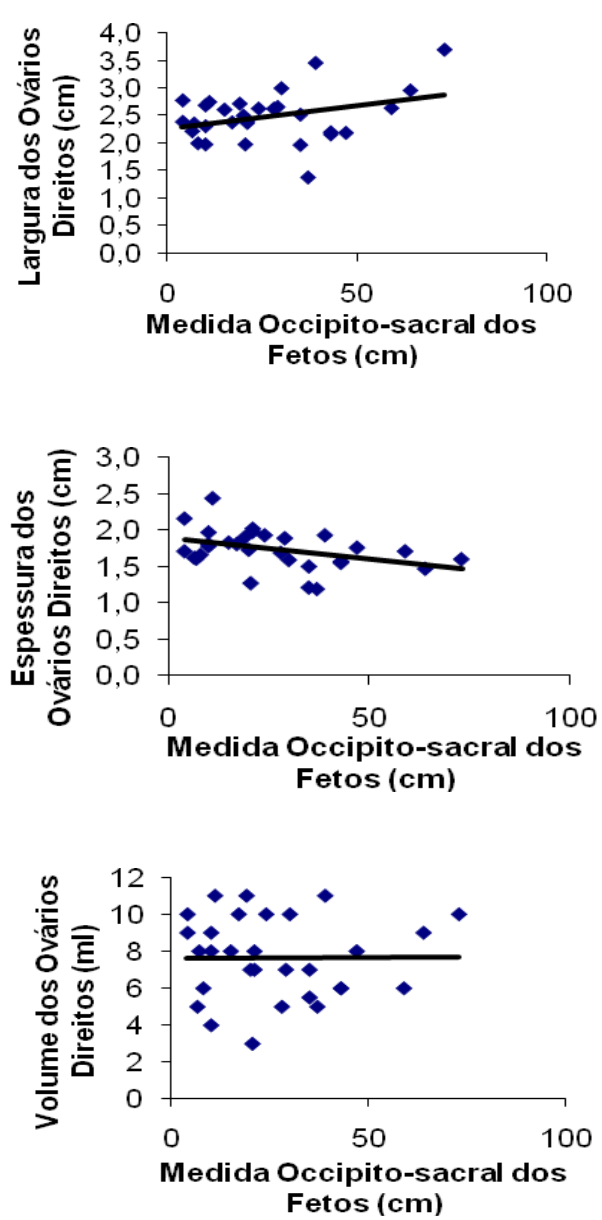

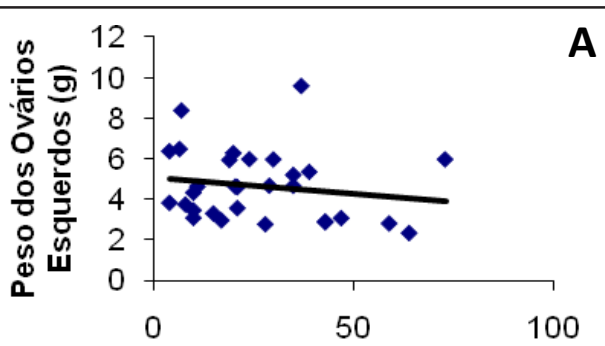

Medida Occipito-sacral dos Fetos (cm)
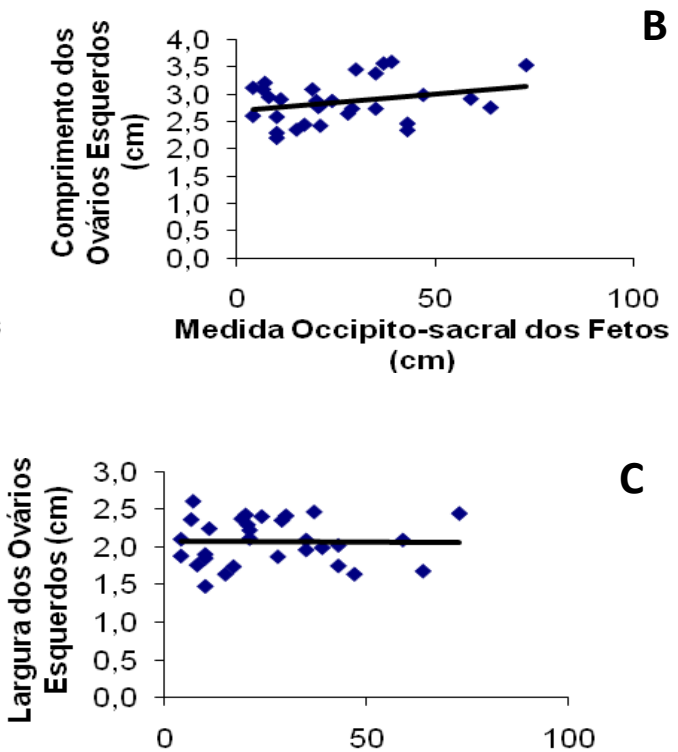

Medida Occipito-sacral dos Fetos (cm)
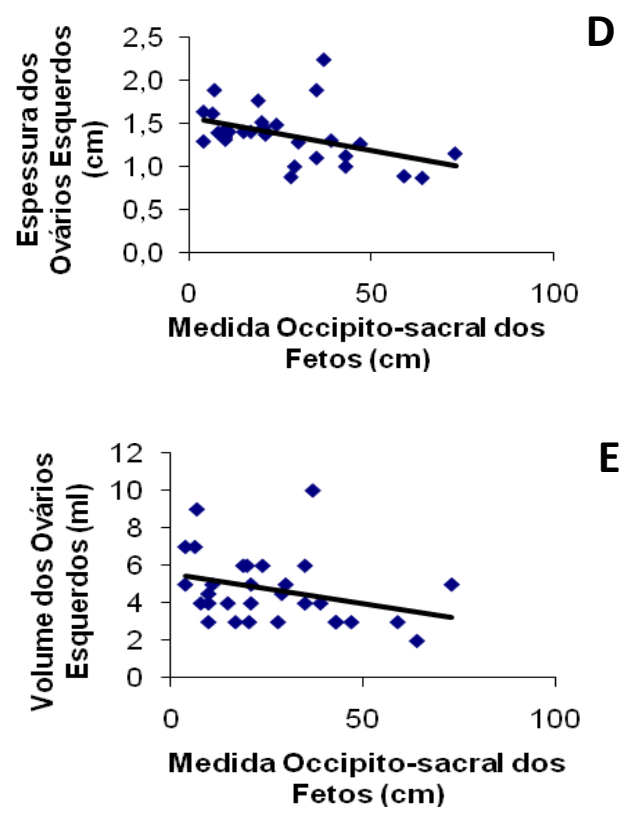
FIGURA 2: Regressão Linear Simples dos pesos (A), comprimentos (B), larguras (C), espessuras (D) e volumes dos corpos lúteos (E).
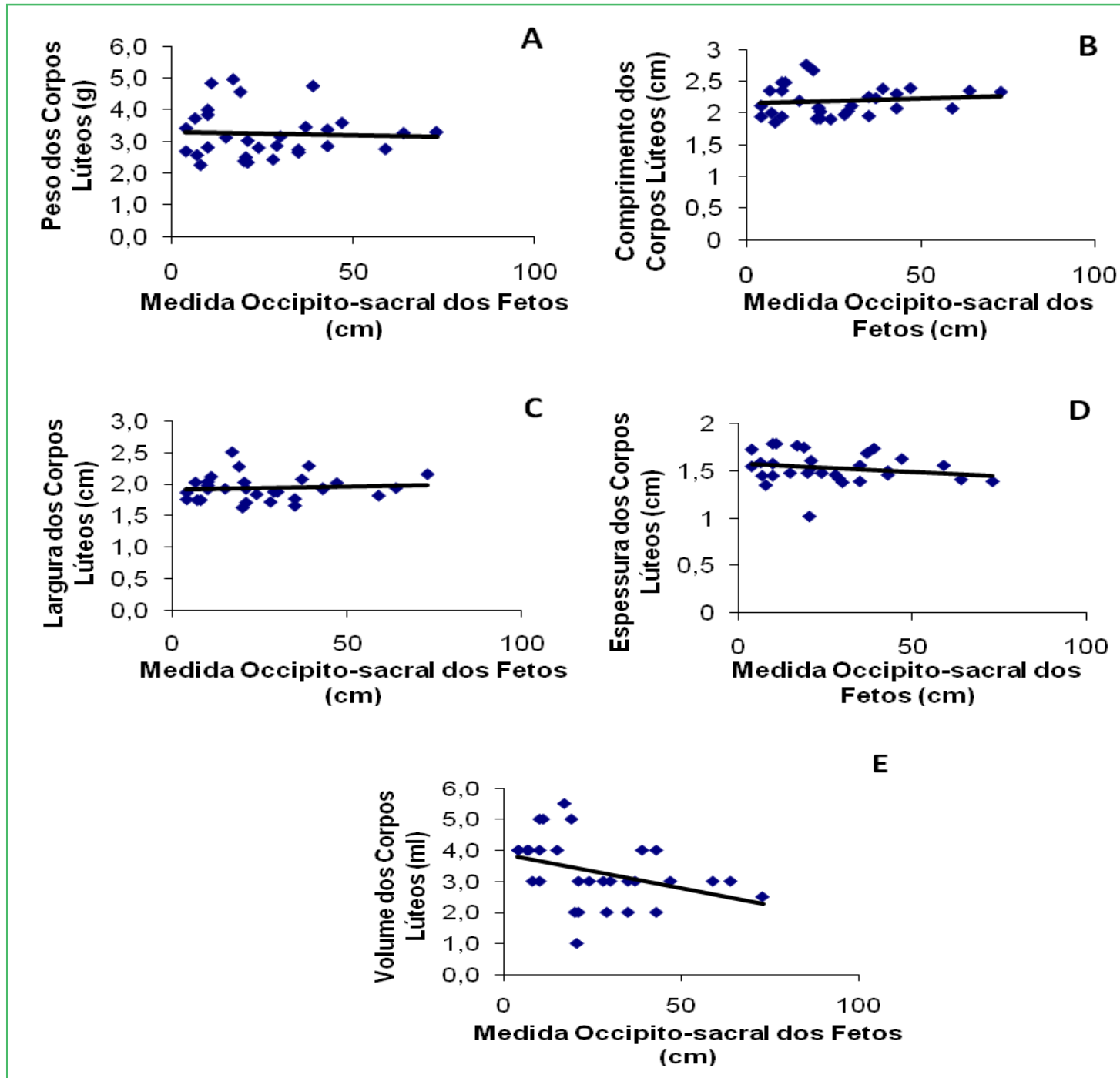

firme, especialmente nas primeiras fases da gestação. O tamanho desta estrutura pode alcançar para estes autores $2,0 \mathrm{~cm}$, dado inferior ao que encontramos nos bovinos Nelore, que foi de $2,20 \mathrm{~cm}$.

Sem particularizar a espécie animal, Romer e Parsons (1985) informam que, na estrutura do ovário, além de tecido intersticial, existem células foliculares relacionadas à manutenção dos óvulos em desenvolvimento, e também na produção de estrógenos. Para estes autores o ovário é responsável pela síntese e secreção de hormônio esteróide quando um óvulo é expelido, determinando neste local a evolução do corpo lúteo, que tende a preencher o folículo esvaziado.
De fato em nosso material encontramos a presença do corpo lúteo de forma constante e marcante na superfície do ovário, evento indicativo de que esta estrutura, independentemente da fase gestacional, é importante na manutenção do feto.

Relativamente aos trabalhos específicos, De Lucia (2002) e Moura (2003) revelam interessantes resultados, porém sem estabelecer nenhuma correlação com o desenvolvimento do feto, mas referem ao comportamento do corpo lúteo. Deste modo, Moura (2003) informa que o peso do corpo lúteo em búfalas aumenta de $605 \mathrm{mg}$ no $3^{\circ}$ dia de gestação para $1885 \mathrm{mg}$ no $13^{\circ}$ dia, mas apresenta a seguir um declínio para 
$1292 \mathrm{mg}$ no $18^{\circ}$ dia. Esta oscilação, conforme os dados do autor, está diretamente relacionada à concentração da progesterona circulante, o que de fato é bastante razoável se entendermos que o papel do corpo lúteo como gerador deste hormônio vai ser substituído gradativamente pela placenta.

De sua parte, Viana et al. (1999) analisam as características morfológicas e funcionais do corpo lúteo durante o ciclo estral em vacas da raça Gir, estabelecendo quatro fases para a caracterização desta estrutura, ou seja, luteogênese, luteojovem, luteomaduro e luteólise. Entretanto este autor não oferece nenhum dado correlacionado ao desenvolvimento fetal. Os nossos achados em relação ao desenvolvimento do corpo lúteo nas diferentes fases gestacionais dos bovinos Nelore, não nos permitem estabelecer uma divisão similar ao destes autores, embora possamos admitir que a evolução do corpo lúteo durante a gestação esta diretamente correlacionada com as diversas fases do desenvolvimento do feto.

Ainda neste sentido, Chacur et al. (2006) esclarecem, de forma semelhante à Nascimento (2003), que os ovários de vacas mestiças apresentam características morfológicas conforme a fase do ciclo estral. Esta morfologia, conforme os autores, pode ser utilizada como parâmetro na avaliação clínica e funcional do ovário. Estes autores informam ainda que devido à presença de corpos lúteos inclusos nas vacas zebus, a palpação retal destas estruturas nem sempre é eficaz para um prognóstico da atividade ovariana luteal cíclica. Como em nossa amostra não encontramos corpos lúteos do tipo incluso, ainda cabe ressaltar nesse sentido a utilização da palpação retal para avaliar estas estruturas.

As informações que obtivemos nos bovinos da raça Nelore permitem estabelecer que o Coeficiente de Correlação de Pearson entre as variáveis dos ovários direitos e desenvolvimento do feto, obteve-se uma correlação significativa para largura e espessura do ovário, onde a primeira foi positiva, ou seja, conforme aumenta o desenvolvimento do feto observou-se também um aumento da largura dos ovários. Já a segunda foi negativa, indicando que conforme aumento o desenvolvimento fetal há uma diminuição da espessura dos ovários, todos com nível de $\mathrm{p}<0,05$.
Para a análise do Coeficiente de Correlação de Pearson entre as variáveis dos ovários esquerdos e desenvolvimento do feto, não foi observada uma correlação significativa para nenhuma das variáveis, nos quais todas não significativas ao nível $p<0,05$. Já que o número de ovários esquerdos com a presença do corpo lúteo foi muito reduzido na amostra realizada, isto pode ter influenciado na análise dos dados na estatística $(p<0,05)$.

Da correlação entre o CL e a MOS, somente o volume teve uma correlação negativa e significativa, indicativo de que conforme há um aumento do tamanho do feto, observa-se uma diminuição do volume do CL, com nível de $\mathrm{p}<0,05$.

Mediante as observações pode-se concluir que houve correlação significativa entre a largura e espessura dos ovários com o desenvolvimento do feto, com a primeira positiva e a segunda negativa. Já com os corpos lúteos houve uma correlação significativa e negativa entre o seu volume e o desenvolvimento do feto.

\section{Agradecimentos}

À Fundação de Apoio à Pesquisa do Estado de São Paulo (FAPESP) Proc. 07/51105-6 (Bolsa de Iniciação Científica do primeiro autor) pelo apoio no desenvolvimento deste projeto.

\section{Referências}

BARONE, R. Anatomie commpareé des mammifères domestiques. Paris: Vigot, 1990. 951 p.

BINELLI, M. Estratégias anti-luteolíticas para a melhora da sobrevivência embrionária em bovinos. In: BARUSELLI, P. S.; MADUREIRA, E. H. (Eds). Controle farmacológico do ciclo estral. São Paulo: USP, 2000. p. 99-114.

CHACUR, M. G. M.; VALENTIM, N. C.; MARTINEZ, A. I. S.; TOSTES, R. A.; KRONKA, S. N. Morfometria de ovários de fêmeas Zebu Bos taurus indicus coletados em matadouro. Acta Scientiae Veterinariae, Porto Alegre, v. 34, n. 1, p. 65-70, 2006.

DE LUCIA, R. F. S. Avaliação e caracterização da fisiologia ovariana e da fertilidade de novilhas Nelore (Bos taurus indicus) de linhagem precoce. 2002. 67 f. Dissertação (Mestrado) - Universidade de São Paulo, São Paulo. 2002.

DYCE, K. M.; SACK, W. O.; WENSING, C. J. G. Tratado de anatomia veterinária. Rio de Janeiro: Guanabara, 1997. 548 p.

FIELDS, M. J.; FIELDS, P. A. Morphological characteristics of 
the bovine corpus luteum during the estrous cycle and pregnancy. Theriogenology, Worburn, v. 45, p. 1295-1325, 1996.

FRANDSON, R. D. Anatomia e fisiologia dos animais domésticos. Rio de Janeiro: Guanabara, 1979. 429 p.

GETTY, R. Sisson/Grossman anatomia dos animais domésticos. v. 1.5 ed. Rio de Janeiro: Guanabara, 1986. 1134 p.

KONIG, H. E.; LIEBICH, H. G. Anatomia dos animais domésticos. 5 ed. Porto Alegre: Artmed, 2011. 787 p.

MOURA, C. E. B. Expressão do VEGF e vascularização do corpo lúteo em búfalos. 2003. 122 f. Dissertação (Mestrado) Universidade de São Paulo, São Paulo. 2003.

NASCIMENTO, A. A.; PINHEIRO, N. L.; SALES, A.; VIANA, J. H. Correlação morfométrica do ovário de fêmeas bovinas em diferentes estádios reprodutivos. Brazilian Journal of Veterinary Research and Animal Science, São Paulo, v. 40, n. 2, p. 126-132, 2003.

NEVES, M. M.; MARQUES JR, A. P.; SANTANA, C. V.; LIMA, F. P. C.; ZAMBRANO, W. J. Características de ovários de fêmeas zebu (Bos taurus indicus) colhidos em abatedouro. Arquivo Brasileiro de Medicina Veterinária e Zootecnia, Belo Horizonte, v. 54, p. 651-654, 2002.
NICKEL, R.; SCHUMMER, A; SEIFERLE, E. The viscera of the domestic mammals. 2. ed. New York: Verlag P. Parey, 1979. 401 p.

ROBERTS, S. J. Veterinary obstetrics and genital diseases (Theriogenology). 3. ed. Ann Arbor: Edwards Brothers, 1986. 776 p.

ROMER, A. S.; PARSONS, T. S. Anatomia comparada dos vertebrados. São Paulo: Atheneu, 1985. 559 p

SAS. SAS ${ }^{\circledR}$ (Statistical Analysis System) User's Guide: Statistics. Version 9.1. 4 ed. Inc., Cary. NC. 2002.

SCHALLER, O. Nomenclatura anatômica veterinária ilustrada. São Paulo: Manole, 1999. 614 p.

SCHWARZE, E.; SCHRÖDER, L. Compêndio de anatomia veterinária. v. 2. Zaragoza: Acribia, 1970. 313 p.

VIANA, J. H. M; FERREIRA, A. M; SÁ, W. F.; CAMARGO, L. S. A. Características morfológicas e funcionais do corpo lúteo durante o ciclo estral em vacas da raça Gir. Arquivo Brasileiro de Medicina Veterinária e Zootecnia, Belo Horizonte, v. 51, p. 251-256, 1999.

ZHENG, J.; FRICKE, P. M.; REYNOLDS, L. P.; REDMER, D. A. Evaluation of growth, cell proliferation, and cell death in bovine corpora lutea throughout the estrous cycle. Biology of Reproduction, New York, v. 51, p. 623-632, 1994. 